\title{
Special Issue on Nano-Biointerface for Biosensing
}

\author{
Cristina Satriano $\mathbb{D}$ \\ Laboratory of Hybrid NanobioInterfaces (NHIL), Department of Chemical Sciences, University of Catania, \\ viale Andrea Doria 6, 95125 Catania, Italy; csatriano@unict.it; Tel.: +39-095-738-5136
}

Received: 17 October 2019; Accepted: 21 October 2019; Published: 24 October 2019

\section{Introduction}

Point-of-care nanobiosensors have tremendous potential to revolutionize the future of personalized nanomedicine, especially for the simultaneous diagnosis and therapy, which takes the name of theranostics. Nanoscale-sized biosensors offer several advantages compared to traditional biosensors, including the enhanced loading of desired biomolecules due to their larger surface area; the multifaceted properties of nanostructured materials, thus the capability for simultaneous measurements of multiple biomarkers; and the increased sensitivity for the detection of a minute biomolecule concentration. With the rapid growth of nanotechnology, an increased interest has focused on the development of engineered nanomaterials suitable for a triggered interaction at the biological interface, for the development of novel sensing devices targeted to analytes ranging from small molecules to proteins to oligonucleotides. In general, functionalized nanomaterials, owing to their unique and tunable electrochemical and optical properties, are very advantageous as immobilization platforms, catalytic tools, and optical and electroactive labels to enhance biosensing characteristics, such as sensitivity, stability, and selectivity [1]. Additionally, the incorporation of multiple nanomaterials into composite films can take advantage of the superior and potentially synergistic properties of each component. Different detection methods, including those based on fluorescence or electrochemistry or surface plasmon resonance or acoustic evanescent waves, have advanced through using new nanomaterials prepared in a wide range of different structures. Based on the design of the nanoplatform, a target recognition can be performed in the biological environment (e.g., towards enzymes, antibodies, proteins, etc.) and the nano-bio interaction can be transduced into amplified readouts. In light of the above, this special issue was introduced to collect the latest research on relevant topics of nanomaterial-based biosensors, and more importantly, to address present challenging issues with the fundamentals of nanomaterials-based biosensors, including the different components of transducers, such as the electrochemical, optical, piezoelectric, thermal and surface plasmon resonance, and their responses at the hybrid biointerface. The contributed articles belong to two broad groups; i.e., (i) optical and electrochemical sensing for cells and bacteria; (ii) nanobiosensors aimed to target polypeptides for an early diagnosis of disease.

\section{Optical and Electrochemical Sensing for Eukaryotic Cells and Bacteria}

The development of a robust sensing system suitable for biomedical screening needs to cross the boundaries of traditional cell labelling approaches based on fluorophores that, often, are phototoxic, not sensitive enough or specific to eukaryotic cell models, and most relevantly, suffer self-quenching or bleaching in the case of prolonged light exposure [2].

E.L. Sciuto et al. in their paper on the Study of a Miniaturizable System for Optical Sensing Application to Human Cells [3], combine the cell labelling by Tris(2,2'bipyridyl)ruthenium(II) $\left(\mathrm{Ru}(\mathrm{bpy})_{3}{ }^{2+}\right)$ fluorophore with the detection by a sensitive and miniaturizable laser beam-photomultiplier based system. The uptake of $\mathrm{Ru}(\mathrm{bpy})_{3}{ }^{2+}$ in human dermal fibroblasts (HDF) or in human colon adenocarcinoma (LoVo) cells was successfully detected by fluorescent time-lapse analysis and confirmed by fluorescence microscopy (FM) and confocal laser scanning microscopy (CLSM). Cells survival 
from the labelling method was checked by MTT, (3-(4,5-Dimethylthiazol-2-yl)-2,5-diphenyltetrazolium bromide, commonly used to detect reductive metabolism in cells for viability, proliferation and cytotoxicity assays), of cells exposed to the fluorophore.

Nanocomposites of spherical gold nanoparticles (AuNPs) and graphene oxide (GO) nanosheets, fabricated and characterized by L.M. Cucci and coworkers in their contribution: A Hybrid Nanoplatform of Graphene Oxide/Nanogold for Plasmonic Sensing and Cellular Applications at the Nanobiointerface [4], have been developed as highly promising nanoplatform for theranostic applications targeted to cancer cells. The plasmonic sensing capability of spherical gold nanoparticles-roughly $10 \mathrm{~nm}$ in the diameter and mostly confined at the GO basal planes, as shown by atomic force microscopy (AFM) analyses-are maintained and enhanced in the hybrid nanoassembly, despite the strong nanoparticle/nanosheet association revealed by nuclear magnetic resonance (NMR) spectroscopy. The GO-AuNP cytotoxicity, as well as their effects on both total and mitochondrial reactive oxygen species (ROS) production in human neuroblastoma cells (SH-SY5Y line), were demonstrated by MTT assays and by experiments with 2,7-dichlorofluorescein (DCF) and MitoSOX, respectively. Laser scanning confocal microscopy (LSM) imaging confirmed the increased uptake of the nanosheet in the GO-AuNP hybrids compared to the GO alone, which lead to mitochondrial dysfunctions, and in turn, the ROS generation.

Electrochemical analysis is based on the redox behavior of the analyte. A variety of electrochemical techniques are available for the detection of biomolecules; however, the development of a sensing platform with high sensitivity and selectivity is still challenging. The innovation introduced by multifunctional nanomaterials has contributed to great advancement of electroanalytical technologies for in vivo and in vitro analyses, especially for point-of-care clinical applications [5].

S. Durairaj and coworkers in their contribution on Nanomaterials-Based Electrochemical Sensors for In Vitro and In Vivo Analyses of Neurotransmitters [6], review the working principles of the most commonly employed electrochemical analysis techniques for the detection of neurotransmitters, with a special focus on the fabrication and development of nanomaterial-based electrochemical sensors. Challenges and future outlook are described for a more efficient detection of neurotransmitters by electrochemical sensors with improved selectivity in real-time analysis and decreased drawbacks in animal models due to biofouling and interfering molecules. The integrated use of membrane-based sensors or combinations of micro dialysis is evoked to reinforce the ongoing research in the design of advanced nanomaterials-based in vivo electrochemical sensors, which may present exciting new avenues in neuroscience, since the understanding of the brain and of central and peripheral nervous system would provide unprecedented insights into the neurochemical basis of behavior and disease.

S.R. Chinnadayyala and coworkers in their article: Electrochemical Impedance Characterization of Cell Growth on Reduced Graphene Oxide-Gold Nanoparticles Electrodeposited on Indium Tin Oxide Electrodes [7], describe the fabrication of reduced graphene oxide-gold nanoparticles (rGO-AuNP) electrodeposited onto a transparent indium tin oxide (ITO) electrode and investigate the feasibility of monitoring HEK293 cell growt by electrochemical impedance, in combination with the microscopic observation of the cells transfected with a green fluorescent protein expression vector. Results demonstrate the high potentiality of the rGO-AuNP/ITO electrode for label-free and real-time impedimetric cell-based assays, since the rGO-AuNP hybrids are biocompatible and induce an increase in cell adherence to the electrode when compared to the bare, AuNP-, or rGO-deposited ITO electrode.

$\mathrm{H}$. Wang and coworkers in their paper on the Development of an Electrochemical Biosensor for Rapid and Effective Detection of Pathogenic Escherichia coli in Licorice Extract [8], describe the development of an aptamer-based electrochemical biosensor and its application in the rapid detection of pathogenic Escherichia coli (E. coli) in licorice extract. For the biosensor fabrication, thiolated capture probes were firstly immobilized on a gold electrode, and then the biotinylated aptamer probes for E. coli were introduced by hybridization with the capture probes. The sensing principle of the system is related to the stronger interaction between the aptamer and the E. coli, causing the dissociation of a part of the biotinylated aptamers from the capture probes in the presence of E. coli. Then, residual biotinylated aptamer probes can quantitatively bind with streptavidin-alkaline phosphatase and 
$\alpha$-naphthyl phosphate; substrates are catalytically hydrolyzed to generate an electrochemical response, recorded by a differential pulse voltammetry. This approach of electrochemical biosensing strategy can be used for developing other biosensors for pathogenic microorganisms and becomes a powerful tool for pathogenic bacteria's detection in the fields of food and drug safety, quality control, clinical diagnostics, and environmental monitoring.

In the manuscript: A Review on Nanoparticles as Boon for Biogas Producers-Nano Fuels and Biosensing Monitoring [9], S. Faisal and coworkers overview the use of nanoparticles as an additive to feed bacteria that break down natural substances in bioenergy production from the biomass. The assessment of anaerobic digestion is based on the continuous monitoring of organic and volatile fatty acids, resulting in the accumulation of intermediates for unsteady progression conditions. Since instantaneous intervention is not possible due to the time between sampling and the availability of the results, acid content is typically only analyzed once or twice per month. To face this problem, biosensors have been devised for the accurate and fast analysis of many compounds. The focus of this manuscript is on the novel notion of dosing ions using modified nanoparticles that can be used to progress up biogas production in oxygen free digestion processes.

\section{Protein and Peptide Nanobiosensors for the Early Diagnosis of Diseases}

A pathological process at an early stage is characterized by the appearance of marker proteins at very low $\left(10^{-15} \mathrm{M}\right.$ and lower) concentrations in blood, and highly-sensitive nanobiosensor systems are required to detect proteins at such low concentrations [10]. Additionally, the composition of biomolecules gathering at a surface is another relevant issue, given the dynamic nature of disease progression. With respect to that, nano-biosensors may offer increased possibilities for the early diagnosis of diseases.

In the manuscript: Influence of Chip Materials on Charge Generation in Flowing Solution in Nanobiosensors [11], Y.D. Ivanov and coworkers demonstrate that the presence of an AFM chip (made of mica and graphite) near the nozzle of the injector supplying an analyte solution into the measuring cell of an AFM-based fishing system (used for the registration of low-abundant proteins in the temperature range corresponding to the physiological temperature of $35^{\circ} \mathrm{C}$ ) causes an increase in charge generation upon the injection of the solution. In the presence of protein at femtomolar concentrations, an increase in the efficiency of charge generation in the injector was demonstrated.

G.Z. Gayda and coworkers in their paper on Metallic Nanoparticles Obtained via "Green" Synthesis as a Platform for Biosensor Construction [12] demonstrate the possibility of developing reagent-less, enzyme-based amperometric biosensors using metallic nanoparticles obtained via green synthesis (gNPs), which have great potential for applications in biotechnology, industry, and medicine as antibacterial agents, fluorescent markers, and carriers for drug delivery, respectively. The bioelectrodes modified with Pd-based gNPs have lower sensitivities to their substrates, but broader linear ranges of their detection and higher storage stabilities than unmodified control electrodes. Moreover, the advantage of the "green" approach is the rapid growth of the yeast cells with a high-yield of pure gNPs surrounded by organic compounds that provides the anti-aggregative effect on metallic gNPs, which can be useful as carriers of enzymes and cells in biosensors and biofuel cells.

In the article on The Characterisation and Quantification of Immobilised Concanavalin A on Quartz Surfaces Based on The Competitive Binding to Glucose and Fluorescent Labelled Dextran [13] authored by T. Bich Hoang and coworkers, a simple protocol for the immobilization of Concanavalin A (Con A) on a quartz substrate, a common material for gravimetric microsensors, was developed. The binding activity of the immobilized Con A was assessed by measuring the intensity from fluorescent labelled dextran (FITC and Alexa 488; with a surface coverage ranging from $1.8 \times 10^{11}$ to $2.1 \times 10^{12}$ immobilized fluorescent molecules per $\mathrm{cm}^{2}$ ) over a glucose concentration range from 0 to $40 \mathrm{mM}$, whereas any structural changes to the surface topography were investigated using AFM. These complementing measurements verified that a change in the fluorescence intensity was accompanied with a change 
in the average surface roughness, and thereby the composition (addition and loss of dextran) at the sample surface.

H. Aldewachi and coworkers in their paper on the Study of the Stability of Functionalized Gold Nanoparticles for the Colorimetric Detection of Dipeptidyl Peptidase IV [14] report on three stabilization strategies of gold nanoparticles and their practical applications for the visual detection of dipeptidyl peptidase IV (DPP-IV), an enzyme of high clinical importance, especially as a diagnostic and prognostic marker of various inflammatory disorders and tumors. The first strategy used the incorporation of a PEGylated sequence with a dithiol anchor to create biorecognition ligands; the other two strategies used a peptide sequence (CALNN) selected from 58 peptide sequences tested for their high stability and resistance to electrolyte-induced Au NP aggregation. DPP-IV/CD26 activity using the three approaches was detected under optimal conditions and only C/G-capped AuNPs showed a response with a color change when incubated with DPP-IV/CD26. C/G-capped AuNPs were less sensitive than the monofunctionalized systems, with a dynamic range of $10^{-30} \mathrm{U} / \mathrm{L}$ but with very high stability in ionic solutions. The developed biofunctionalized assay was successfully evaluated by gel electrophoresis, zeta potential, and hydrodynamic radius measurements and tested for the assay of DPP-IV activity.

Finally, in the review: Biosensor Applications of Electrodeposited Nanostructures [15], the author K.J. Stine traces an overview on the area of developing biosensors based on electrodeposited nanostructures, which has many possible applications to the detection of small molecules, metabolites, protein antigens, oligonucleotides, and other targets, including bacteria and viruses. The variety of substrate electrodes and preparation conditions can produce a vast range of structures. Many additional targets of high interest for the development of diagnostics, such as glycoproteins, circulating tumor cells, circulating oligonucleotides, microRNAs, and content in microvesicles remain unexplored.

Funding: This research received no external funding.

Conflicts of Interest: The author declares no conflict of interest.

\section{References}

1. Di Pietro, P.; Strano, G.; Zuccarello, L.; Satriano, C. Gold and Silver Nanoparticles for Applications in Theranostics. Curr. Top. Med. Chem. 2016, 16, 3069-3102. [CrossRef] [PubMed]

2. Trusso Sfrazzetto, G.; Satriano, C.; Tomaselli, G.A.; Rizzarelli, E. Synthetic fluorescent probes to map metallostasis and intracellular fate of zinc and copper. Coord. Chem. Rev. 2016, 311, 125-167. [CrossRef]

3. Sciuto, E.; Villaggio, G.; Santangelo, M.; Laudani, S.; Federico, C.; Saccone, S.; Sinatra, F.; Libertino, S. Study of a Miniaturizable System for Optical Sensing Application to Human Cells. Appl. Sci. 2019, 9, 975. [CrossRef]

4. Cucci, L.; Naletova, I.; Consiglio, G.; Satriano, C. A Hybrid Nanoplatform of Graphene Oxide/Nanogold for Plasmonic Sensing and Cellular Applications at the Nanobiointerface. Appl. Sci. 2019, 9, 676. [CrossRef]

5. Kaushik, A.; Mujawar, M. Point of Care Sensing Devices: Better Care for Everyone. Sensors 2018, 18, 4303. [CrossRef] [PubMed]

6. Durairaj, S.; Sidhureddy, B.; Cirone, J.; Chen, A. Nanomaterials-Based Electrochemical Sensors for In Vitro and In Vivo Analyses of Neurotransmitters. Appl. Sci. 2018, 8, 1504. [CrossRef]

7. Chinnadayyala, S.; Park, J.; Choi, Y.; Han, J.-H.; Yagati, A.; Cho, S. Electrochemical Impedance Characterization of Cell Growth on Reduced Graphene Oxide-Gold Nanoparticles Electrodeposited on Indium Tin Oxide Electrodes. Appl. Sci. 2019, 9, 326. [CrossRef]

8. Wang, H.; Zhao, Y.; Bie, S.; Suo, T.; Jia, G.; Liu, B.; Ye, R.; Li, Z. Development of an Electrochemical Biosensor for Rapid and Effective Detection of Pathogenic Escherichia coli in Licorice Extract. Appl. Sci. 2019, 9, 295. [CrossRef]

9. Faisal, S.; Yusuf Hafeez, F.; Zafar, Y.; Majeed, S.; Leng, X.; Zhao, S.; Saif, I.; Malik, K.; Li, X. A Review on Nanoparticles as Boon for Biogas Producers-Nano Fuels and Biosensing Monitoring. Appl. Sci. 2018, 9, 59. [CrossRef]

10. Mehrotra, P.; Chatterjee, B.; Sen, S. EM-Wave Biosensors: A Review of RF, Microwave, mm-Wave and Optical Sensing. Sensors 2019, 19, 1013. [CrossRef] [PubMed] 
11. Ivanov, Y.; Kozlov, A.; Galiullin, R.; Tatur, V.; Ivanova, N.; Ziborov, V. Influence of Chip Materials on Charge Generation in Flowing Solution in Nanobiosensors. Appl. Sci. 2019, 9, 671. [CrossRef]

12. Gayda, G.; Demkiv, O.; Stasyuk, N.; Serkiz, R.; Lootsik, M.; Errachid, A.; Gonchar, M.; Nisnevitch, M. Metallic Nanoparticles Obtained via "Green" Synthesis as a Platform for Biosensor Construction. Appl. Sci. 2019, 9, 720. [CrossRef]

13. Hoang, T.; Stokke, B.; Hanke, U.; Johannessen, A.; Johannessen, E. The Characterisation and Quantification of Immobilised Concanavalin A on Quartz Surfaces Based on The Competitive Binding to Glucose and Fluorescent Labelled Dextran. Appl. Sci. 2019, 9, 318. [CrossRef]

14. Aldewachi, H.; Woodroofe, N.; Gardiner, P. Study of the Stability of Functionalized Gold Nanoparticles for the Colorimetric Detection of Dipeptidyl Peptidase IV. Appl. Sci. 2018, 8, 2598. [CrossRef]

15. Stine, K.J. Biosensor Applications of Electrodeposited Nanostructures. Appl. Sci. 2019, 9, 797. [CrossRef]

(C) 2019 by the author. Licensee MDPI, Basel, Switzerland. This article is an open access article distributed under the terms and conditions of the Creative Commons Attribution (CC BY) license (http://creativecommons.org/licenses/by/4.0/). 